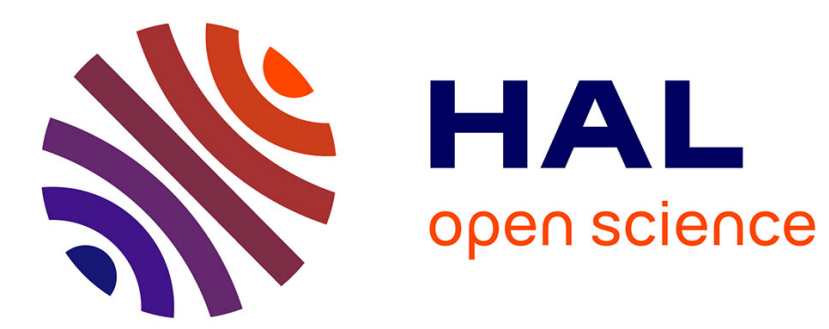

\title{
La cognition animale et l'éthique
}

Joëlle Proust

\section{- To cite this version:}

Joëlle Proust. La cognition animale et l'éthique. Le Debat , 2000, 108, pp.175-183. ijn_00139418

\section{HAL Id: ijn_00139418 \\ https://hal.science/ijn_00139418}

Submitted on 30 Mar 2007

HAL is a multi-disciplinary open access archive for the deposit and dissemination of scientific research documents, whether they are published or not. The documents may come from teaching and research institutions in France or abroad, or from public or private research centers.
L'archive ouverte pluridisciplinaire HAL, est destinée au dépôt et à la diffusion de documents scientifiques de niveau recherche, publiés ou non, émanant des établissements d'enseignement et de recherche français ou étrangers, des laboratoires publics ou privés. 
La cognition animale et l'éthique

(2000), Le Débat, 108, 175-183.

Joëlle Proust

Les primates non humains font-ils partie de la communauté morale, c'est-à-dire des individus à l'égard desquels se pose la question de l'obligation morale : s'ils ne sont pas agents moraux, peuvent-ils du moins être reconnus comme patients moraux, c'est-à-dire comme cibles d'un comportement moralement évaluable ? La tradition avait une réponse à cette question : la raison et la conscience de soi constituent les conditions de la reconnaissance d'un statut de patient moral "à part entière". La question que pose l'article de Paola Cavalieri est non pas de savoir si ces conditions sont véritablement nécessaires pour juger de l'appartenance à la communauté morale, mais si la tradition philosophique est justifiée en affirmant que seul Homo sapiens sapiens les satisfait.

La stratégie argumentative de l'auteur en faveur du statut moral des animaux non-humains s'inscrit à l'intérieur d'une problématique particulière, selon laquelle le statut de patient moral dépend de la possession de caractéristiques intrinsèques, telles que la maîtrise de capacités langagières ou communicationnelles, la conscience de soi et la capacité de raisonner. Il aurait également été possible de soutenir que ce même statut des animaux se justifie non par les propriétés de leur esprit ou de leur expérience, mais par leur simple dignité d'êtres vivants, c'est-àdire en invoquant leur valeur inhérente ${ }^{1}$ de créatures sensibles. C'est d'ailleurs précisément en tant que l'auteur choisit la perspective de la valeur intrinsèque qu'elle délimite son champ d'argumentation : les grands primates paraissent intuitivement plus proches de l'homme qu'aucune autre espèce animale. Tandis que la valeur intrinsèque est susceptible de varier selon la nature des capacités de connaissance et d'action des individus évalués, la valeur inhérente est égale entre tous les êtres qui en sont munis, et n'inviterait pas aussi aisément une stratégie comparative.

Paola Cavalieri fait valoir trois types de raisons à l'appui de la reconnaissance du statut de patient moral aux grands singes. L'argument évolutionniste consiste à avancer qu'il n'existe pas de solution de continuité dans la lignée des espèces, et que de surcroît "il n'existe vraisemblablement pas de capacités proprement

\footnotetext{
${ }^{1}$ Sur cette distinction, cf. T. Regan, The case for Animal Rights, Londres, Routledge \& Kegan Paul, (1983).; J.-Y. Goffi, Le Philosophe et ses animaux, Du Statut éthique de l'animal, Nîmes, Jacqueline Chambon, (1994).
} 
humaines". Cet argument évolutionniste vise à poser les jalons en vue d'établir la plausibilité de la thèse défendue, à savoir qu'une valeur intrinsèque globalement identique doit être reconnue à tous les primates, qu'ils soient ou non humains. L'argument individualiste vise à bloquer un point de vue non intrinsèque en matière de statut moral : il conteste la valeur des principes en vertu desquels on se propose d'étendre les conditions de la reconnaissance morale aux individus "non paradigmatiques", c'est-à-dire ne présentant pas les conditions nécessaires de l'appartenance à l'espèce des sujets moraux. C'est en particulier souvent en s'appuyant sur la force de l'affiliation que l'on choisit de faire bénéficier du statut de patient moral non seulement les sujets raisonnables et conscients, mais aussi leurs apparentés. Si l'on suit ce type de principe, exception ne peut être faite aux conditions de la reconnaissance morale qu'en faveur d'êtres humains, sans qu'aucune justification proprement morale, de l'avis de l'auteur, ne soit donnée de cette exception. Enfin selon l'argument factuel, l'existence de capacités que l'on pourrait qualifier de "protorationnelles" chez les grands primates justifie qu'on leur accorde le bénéfice d'une protection moralement fondée. C'est aux premier et le troisième de ces arguments que nous nous intéresserons dans ce qui suit.

\section{Statut moral et évolution des espèces}

Paola Cavalieri a raison de souligner que la biologie comparative postdarwinienne nous oblige à admettre que, de l'animal non-humain à l'homme, la distance n'est pas si grande qu'il a longtemps semblé. S'il a le mérite de rompre avec une conception anthropocentriste ignorante de l'histoire naturelle, ce type d'argument est notoirement difficile à manier, du fait qu'une fois la continuité dans la nature reconnue, on perd le moyen d'y instaurer la coupure que requiert le statut d'être moral. Si l'on fait de la parenté phylogénétique une garantie de ressemblance, toutes les espèces méritent de proche en proche d'être considérées comme des patients moraux, fourmis et paramécies comprises. S'il n'existe pas de fossé ontologique entre l'homme et les autres animaux, il n'existe pas non plus de raison de distinguer un être moral en vertu de son espèce.

On peut toutefois être continuiste, et admettre la légitimité d'une enquête comparative enracinée dans les thèses évolutionnistes, sans être entraîné sur la pente glissante des parentés. Il faut alors assortir la comparaison d'une méthode de validation théoriquement fondée ; à quelles contraintes l'analyse comparative des biologistes évolutionnistes se soumet-elle en matière d'identification de 
similitudes ? Un comparatiste tel que Plotkin² distingue trois manières de procéder qui déterminent chacune un choix du type de populations animales pertinent et conduisent à l'identification de traits communs particuliers. On peut rapprocher deux espèces différentes en s'appuyant sur leur homologie; en d'autres termes, les traits phénotypiques des deux espèces présentent certaines ressemblances parce qu'elles ont un ancêtre commun qui a eu l'attribut précurseur des traits en question (On dit que ces traits sont homologues). C'est précisément le cas de l'homme et du chimpanzé : ils partagent des traits phénotypiques en raison de la divergence phylogénétique qui leur a permis d'évoluer en parallèle depuis quatre millions et demie d'années -- peu de temps à l'échelle de l'évolution des espèces. Mais on peut aussi comparer des espèces soit de manière anagénétique, en remarquant les progrès évolutifs qui marquent la succession des espèces, soit de manière analogique, en observant les propriétés voisines qui se rencontrent dans des espèces non reliées entre elles si elles vivent dans des conditions écologiques semblables. Comme Plotkin le souligne, les diverses méthodes comparatives donnent des résultats différents : une comparaison homologique comparera l'homme au singe ; une comparaison anagénétique, l'homme au dauphin, une comparaison analogique, l'homme au loup. Même si les traits homologues sont parfois intuitivement plus saillants pour l'oeil non exercé, les ressemblances anagénétiques et analogiques sont au moins aussi éclairantes pour comprendre les relations entre les structures fonctionnelles d'espèces différentes.

Il n'y a donc pas seulement une leçon historique à tirer de l'évolution des espèces. L'existence de convergences morphologiques ou fonctionnelles entre des espèces sans ancêtre commun, - comme celle qui existe entre l'oeil du céphalopode et celui du vertébré, permet de supposer que la ressemblance cognitive n'est pas cantonnée à l'intérieur d'un arbre phylogénétique. Pourquoi prendre le chimpanzé comme cible de la réflexion morale, plutôt que le dauphin ou le perroquet? Le choix non réfléchi de la comparaison homologique (hommechimpanzé) ne serait-il pas l'indice de la persistance du point de vue 'espéciste' au sein de la critique dont il fait l'objet?

\section{Les faits}

\footnotetext{
2 Cf. H.C. Plotkin, The function of learning and corss-species comparison, in G.C.L. Davey (dir.), Animal Models of Human Behavior, Chichester, John Wiley and Sons, (1983), 117-134.
} 
On n'en voudra pas à l'auteur d'affirmer qu'lil n'existe probablement pas de capacités proprement humaines", tant cette affirmation vaut plutôt par sa valeur de provocation que pour son sens littéral, vite pris en défaut. Les contre-exemples abondent. Indépendamment de la maîtrise du langage verbal conversationnel, dont il faut persister à reconnaître qu'il est le propre de l'homme, on pourrait citer de très nombreuses capacités comme l'écriture, l'agriculture, l'activité mathématique, le sport et le jeu à règles conventionnelles, la recherche théorique, l'usage d'outils variés et adaptés, le débat social, la planification concertée, et, bien entendu, l'existence d'institutions et de droit public. On ne trouve pas, chez les autres primates, de capacité de coordination sociale et de réglement des conflits permettant de préciser contractuellement, a parte ante, les situations futures des divers individus engagés dans une interaction. On ne trouve pas non plus de capacités mémorielles liées à l'effectuation de tâches qui, de près ou de loin, puissent être comparées à ce que la maîtrise de l'écriture et le contrôle de ses propres états internes permettent à homo sapiens d'accomplir. Enfin, on ne trouve pas la capacité d'expliciter ses propres raisonnements et de découvrir la nature de la divergence entre les pensées d'autrui et les siennes propres. Cette dernière compétence, liée à la compréhension d'autrui et de ses états mentaux, paraît en dépit d'un optimisme initial des chercheurs continuistes, être finalement le terrain réservé de l'espèce humaine.

Ce que veut probablement dire l'auteur au-delà de la formule provocante, c'est que l'on trouve chez les primates non humains des précurseurs de ces capacités proprement humaines. Par exemple, le chimpanzé suit la direction du regard de ses congénères : il n'en tire pas l'idée que l'autre ait vu un objet ou connaisse un état de chose ; il se borne à utiliser la direction du regard de manière pratique et non réfléchie, comme l'indice d'un élément à consommer ou à fuir. Ce comportement de détection-imitation du regard préfigure ou plus exactement rend possible l'attention conjointe que l'on trouve chez le bébé humain dès l'âge de neuf mois. En ce sens, on peut sans doute voir dans ce comportement de poursuite $\mathrm{du}$ regard un trait homologue des primates. Toutefois, la compréhension d'autrui comme sujet d'expériences et "agent de contemplation" suppose davantage que le simple mécanisme de détection du regard. Il est remarquable à cet égard que les primates non-humains, à la différence des enfants humains, ne produisent pas spontanément de geste ostensif. Quoiqu'on puisse leur apprendre à montrer du doigt, ils utilisent le geste pour obtenir d'autrui un 
objet convoité, et non pour montrer un objet à autrui à seule fin de le contempler ${ }^{3}$.

Il faut ainsi souligner la différence fondamentale entre l'exploitation d'une source d'information et la représentation de cette information comme ayant une source mentale. Même si le chimpanzé tire parti du dispositif inné par lequel il suit le regard de ses congénères, il ne sait pas que ses congénères ont des états mentaux propres, liés à leur expérience acquise. Quoiqu'il puisse efficacement coordonner son action avec les membres de son groupe, il ne dispose pas de la capacité d'attention conjointe à laquelle les humains ont accès. L'attention conjointe suppose en effet que l'initiateur de la communication ait l'intention de faire reconnaître par autrui les propriétés d'un objet ou d'un événement qui sont au centre de son expérience, ainsi que l'intention de poursuivre l'expérience de manière conjointe en vertu de la première intention. Cette articulation entre contenus intentionnels semble jouer un rôle capital dans la communication de type humain. Elle fait entièrement défaut chez les primates non humains vivant dans leur environnement naturel ${ }^{4}$.

On ne peut donc pas suivre Paola Cavalieri lorsqu'elle affirme que, chez les orang-outans, "la transmission culturelle se fait par un véritable enseignement". Enseigner un savoir-faire suppose que l'enseignant se représente l'état d'ignorance initial de son élève et l'état-cible de savoir qu'il souhaite obtenir à l'issue de l'enseignement. Dans la mesure où les primates ne se représentent pas l'état des connaissances des autres individus ${ }^{5}$, on estime généralement qu'il est impropre de parler d'enseignement à propos de comportements qui peuvent recevoir une interprétation plus directe. Par exemple, une mère chimpanzé qui

\footnotetext{
${ }^{3}$ Les primatologues distinguent le cas des animaux élevés par l'homme de ceux qui vivent dans leur environnement naturel. De nombreux comportements nouveaux apparaissent dans le premier groupe, qui procèdent de l'acculturation humaine, mais ne constituent pas le répertoire normal des capacités des animaux du second groupe. On ne peut donc dériver du cas d'individus remarquables du premier groupe, tels que l'orang-outan Chantek, le gorille Koko, les chimpanzés Kanzi ou Sarah, les propriétés naturelles des individus du second groupe (cf. J.C. Call \& M. Tomasello, "The effect of humans on the cognitive development of apes", in A.R. Russon, K.A. Bard \& S.T. Parker, (dirs.), Reaching into thought : the minds of the great apes, (New York: Cambridge University Press, 1996),371-403.

${ }^{4}$ Les grands singes élevés par l'homme peuvent dans une certaine mesure apprendre à utiliser les moyens humains de capture sociale de l'attention et à utiliser dans certaines circonstances des pointages ostensifs. L'interprétation cognitive de ces comportements est encore débattue.

${ }^{5}$ Cf. D. Povinelli, "Chimpanzee theory of mind ? the long road to strong inference", in P. Carruthers \& P.K. Smith, (eds.), Theories of theories of mind, (Cambridge: Cambridge University Press, 1996): 293-329.
} 
retire des mains de son enfant une plante empoisonnée lui enseigne-t-elle la nocivité de la plante ou l'empêche-t-elle de s'empoisonner ${ }^{6}$ ?

Certes les primatologues de terrain observent l'existence de cultures spécifiques à une communauté d'animaux ( par "culture", on entend la possession de savoirfaire locaux relatifs à la préparation de la nourriture). Par exemple, les macaques japonais de Koshima ont appris en quatre ans à laver leurs patates douces pour les débarrasser de la terre après qu'un seul individu ait inventé cette technique. Mais ce cas relève-t-il de l'enseignement, pratique intentionnelle dirigée vers autrui ? Rien dans le comportement des macaques ayant acquis la technique de nettoyage n'indique le moindre souci pédagogique. La lenteur de l'acquisition tend à montrer que les animaux n'acquièrent l'innovation ni par enseignement délibéré, ni même par imitation. Là où l'observateur non averti croit voir le fruit d'un enseignement, l'éthologue repère un phénomène $d$ '"intensification $d u$ stimulus" : le simple voisinage spatial entre un membre du groupe et l'objet-cible élève l'intérêt des congénères pour ce type d'objet et suscite chez eux des tentatives d'utilisation ${ }^{7}$. C'est ce phénomène qui explique la contagion de comportements nouveaux comme le décapsulage des bouteilles de lait par les mésanges dans l'Angleterre des années cinquante. Ces oiseaux ne savent pas imiter un comportement instrumental, c'est-à-dire ne peuvent pas le reproduire après l'avoir observé. Mais ils peuvent acquérir une motivation particulière pour agir par essais et erreurs sur une portion de l'espace qui a été fructueusement exploitée par leurs congénères.

Ce qui vaut de l'attention conjointe vaut d'autres types de comportements de régulation sociale, tels que les gestes d'apaisement ou de réconciliation qui sont indubitablement une condition de possibilité de la coordination sociale telle qu'elle existe dans les groupes humains. On ne peut dans tous cas cas conclure de l'existence d'un précurseur à l'absence de saut qualitatif. Méconnaître le saut qualitatif que représente la possession d'un langage de communication, avec tous les avantages éducationnels et mémoriels qu'il procure, relève d'un effort désespéré pour oublier l'extraordinaire inégalité dans l'adaptation sociale entre l'espèce humaine et les pongidés.

\footnotetext{
6 Comme le remarquent les primatologues, il est impossible de parvenir à une conclusion argumentée sans connaître la fréquence de base des comportements considérés. La saine méthodologie suggère dans ce type de cas d'appliquer à titre conservatoire, et en l'attente de données probantes, l'explication la plus compatible avec l'ensemble des faits, selon laquelle les grands singes n'ont pas la capacité de se représenter en tant que tels les états mentaux

${ }^{7}$ Cf. A. Whiten, A. \& R. Ham, "On the nature and evolution of imitation in the animal kingdom : reappraisal of a century of research", Advances in the Study of Behavior, 21 (1992): 239283 ; M. Tomasello, M. Davis-Dasilva, L. Camak \& K. Bard, "Observational learning of tool-use by young chimpanzees ", Human Evolution 2, (1987): 175-83.
} 


\section{La compétence langagière des grands singes}

Paola Cavalieri présente les primates anthropoïdes comme de véritables virtuoses de la communication symbolique. Ils auraient "mis au point un vocabulaire de centaines de termes". Ils les associeraient "d'une manière qui répond aux critères fondamentaux pour que lui soit reconnue une valeur grammaticale". A ma connaissance, ces deux affirmations ne sont pas fondées. Les animaux à qui l'homme enseigne un langage de lexigrammes ont besoin de milliers d'essais avant d'acquérir leur premier "mot". En aucun cas les animaux n'inventent de mots pour leurs besoins communicationnels. Et, de même qu'ils ne font pas de gestes dont le but serait simplement d'informer autrui, ils n'utilisent pas les mots pour rapporter des faits, mais pour exprimer des désirs, c'est-à-dire contrôler leurs interlocuteurs humains. Kanzi, par exemple, utilise typiquement les mots pour demander de la nourriture ("Cacahuète çà"), du toilettage ("gratter mordre") ou des jeux ("garder ballon").

La reconnaissance d'une "valeur grammaticale" aux productions symboliques des primates peut évidemment donner lieu à une certaine latitude interprétative, selon que l'on impose des conditions strictes à l'évaluation ou que l'on applique un principe de charité illimité, comme c'est souvent le cas. Si l'on s'en tient aux synthèses les plus modérées ${ }^{8}$, la "syntaxe" qu'un chimpanzé peut maîtriser se limite à l'association ordonnée de deux mots ${ }^{9}$. Ce primate paraît d'ailleurs avoir de meilleures performances pour comprendre l'information syntaxique qui est lui est fournie que pour produire des énoncés syntaxiquement structurés ${ }^{10}$. Cette fluctuation dans la production reflète une inaptitude plus fondamentale, qui pourrait servir de critère de non-maîtrise d'une langue : le primate non-humain le plus rompu à l'expression verbale ne peut pas reconnaître qu'une suite particulière de signes est syntaxiquement mal formée. Or comment parler du respect de la valeur grammaticale d'une phrase si l'animal n'a aucun moyen de se représenter, serait-ce tacitement, l'existence d'une règle, et donc ne peut pas distinguer une séquence de symboles bien formée d'une phrase incorrecte?

L'esprit du chimpanzé n'est pas modelé par les exigences de l'interaction avec les hommes, en laboratoire ou dans une famille humaine : ses capacités cognitives ne répondent pas aux exigences d'une vie sociale langagière. Il n'en est que plus

\footnotetext{
${ }^{8}$ Comme celle de Richard Byrne, dans The Thinking Ape, Oxford, Oxford University Press, 1995.

${ }^{9}$ Trois mots dans le cas exceptionnel de Sarah, le chimpanzé vedette de Premack. Cf. D. et A.J. Premack, L'esprit de Sarah, Paris, Fayard, 1984.

10 Cf. les exemples de productions rapportées dans E.S. Savage-Rumbaugh \& D.M. Rumbaugh, The emergence of language, in K.R. Gibson \& T. Imgold (dirs.), Tools, language and Cognition in Human Evolution, Cambridge, Cambridge University Press, 1993, 86-108.
} 
remarquable qu'il parvienne à apprendre au prix d'une vie entière de travail, à répondre à des tâches langagières sur la base de ses capacités d'apprentissage associatif. Encore n'est-ce le cas que de quelques individus particulièrement doués, comme la Sarah de Premack ou le Kanzi des Rumbaugh. Il n'est donc pas étonnant que la performance linguistique à laquelle parvient un primate entraîné de 8 ans ne dépasse pas le niveau d'un enfant humain de 2 ans, lequel apprend la langue sans effort, et sans qu'un entraînement particulier doive être entrepris. Que la compétence langagière humaine ait été directement sélectionnée par l'évolution ${ }^{11}$ ou soit le résultat fortuit de capacités d'apprentissage plus générales, il est aujourd'hui clairement établi que les primates non-humains n'en sont pas pourvus, et achoppent pour cette raison sur les exigences de compositionalité d'un usage productif de la langue.

\section{La tromperie tactique et la conscience de soi}

Les capacités mentales dont jouissent naturellement les grands singes, indépendamment d'une vie captive en interaction avec l'homme, offrent des arguments séduisants aux partisans de l'extension du statut de patient moral aux animaux phylogénétiquement proches de l'homme. Là encore, la tentation permanente consiste à identifier les comportements des primates aux comportements similaires chez l'homme, en suivant en cela le schéma assimilateur propre à la psychologie ordinaire de l'espèce humaine. Mais en anthropomorphisant la description de ces comportements, on en manque la spécificité cognitive et l'on méconnaît la variété des processus qui les sous-tendent dans le monde animal.

Ce qu'on appelle "tromperie tactique" forme un parfait exemple de cette erreur, lourde de conséquence pour le propos éthique qui est ici défendu. La tromperie tactique recouvre l'ensemble des cas où il semble qu'un animal mente intentionnellement à ses congénères pour des buts privés, en modifiant les signaux qu'il donne de manière à tirer parti d'une situation qu'il est seul à connaître. Les cas de tromperie tactique abondent dans les groupes de primates. Un enfant chimpanzé peut, par ses cris, prétendre être attaqué par un adulte de manière à ce que sa mère chasse ce dernier et laisse l'enfant profiter seul d'un butin. Une femelle peut réprimer ses cris de jouissance lorsqu'elle s'accouple dans les fourrés avec un mâle non-dominant. Un chimpanzé peut faire semblant de n'avoir pas remarqué un buisson couvert de baies pour y revenir quand le

11 Sur ce point, voir S. Pinker \& P. Bloom, Natural language and natural selection, in J.H. Barkow, L. Cosmides \& J. Tooby, The Adapted Mind, Oxford, Oxford University Press, 1992, 451493. 
groupe sera passé. Après avoir, pendant un temps, conclu que les primates nonhumains peuvent mentir, c'est-à-dire se comporter délibérément de manière à produire chez autrui des croyances fausses, les chercheurs admettent aujourd'hui que ces comportements peuvent s'expliquer tout autrement, c'est-à-dire par un apprentissage des comportements efficaces dans une situation donnée. Il n'est pas nécessaire, pour produire les résultats attendus, de savoir que ses congénères agissent sur la base de leurs croyances. Il suffit simplement de découvrir le type d'actions à faire ou à ne pas faire dans telle ou telle circonstance ${ }^{12}$.

Ce que nous savons aujourd'hui de l'esprit des singes, c'est que ces animaux sont capables de catégoriser le monde en fonction d'objets et d'événements pertinents pour leur survie, et de stocker en mémoire un grand nombre d'épisodes de leur expérience passée. Ils peuvent ainsi prédire dans une certaine mesure les états du monde, et construire une carte mentale de leur territoire pour orienter leur recherche de nourriture de manière informée ${ }^{13}$. Les grands primates se représentent le monde physique d'une manière voisine de celle d'un sujet humain qui n'aurait pas reçu de formation scientifique ni n'aurait hérité de son groupe une théorie dite naïve. En revanche, ils n'ont sans doute pas du tout la même façon de se représenter l'information mentale, faute de pouvoir former une théorie des états mentaux, ou de pouvoir conjoindre dans la même opération de pensée une représentation factuelle à la représentation d'une situation simplement possible ou entièrement imaginaire ${ }^{14}$. Ce qui vaut de la compréhension d'autrui vaut aussi de la représentation de soi-même. Il est avéré que les grands singes sont capables de faire référence à eux-mêmes lorsqu'ils ont été entraînés à l'usage de symboles. En outre, à la différence des petits singes, la plupart des chimpanzés et des orang-outans adultes ( mais non les gorilles) réussissent à se reconnaître dans un miroir $^{15}$. Mais on ne peut, sur la foi de ces maigres indications, affirmer que les primates non-humains ont un sens de l'identité personnelle approchant du nôtre. Dépourvus de concepts mentaux, incapables de faire des inférences utilisant les dispositions mentales, et de surcroît libérés de la tâche de construire et de raconter une biographie socialement appréciée - à la mode humaine - les primates non-humains ont sans aucun doute une manière bien à eux de concevoir l'identité des individus, sur la base de

\footnotetext{
12 Quand un pluvier "prétend" avoir l'aile brisée pour éloigner le prédateur de sa nichée, il ne se représente pas non plus l'état d'esprit de l'attaquant. Il fait ce que la situation exige, en vertu d'une manifestation motrice innée déclenchée par la représentation de cette situation.

13 Voir J. Vauclair, L'intelligence de l'animal, Paris, Seuil, 1992.

${ }^{14}$ Cf. J. Proust, "Mindreading in non-human primates", Philosophical Topics, 1999 (à paraître).

15 Cf. Taylor-Parker, S., Mitchell, R.W. \& Boccia, M.L., Self-Awareness in Animals and Humans, Cambridge, Cambridge University Press, 1994.
} 
caractéristiques comportementales et de dispositions saillantes (âge, rang dans le groupe, aptitude à réagir agressivement, etc.). Mais il faut rappeler que cette capacité de distinguer individuellement ses congénères s'applique à de nombreuses espèces sociales, y compris les poules.

\section{La valeur intrinsèque des animaux pourvus d'un esprit}

Inutile de poursuivre l'inventaire comparatif : une fois admis que des différences cognitives importantes existent entre Homo sapiens et les autres primates, reste à se demander dans quelle mesure elles sont moralement pertinentes, ce qui conduit rétrospectivement à s'interroger sur la portée morale des ressemblances. L'une des ambiguités de la démonstration du co-auteur du Great Ape's Project est que l'on ne sait pas comment comprendre l'intérêt qu'elle manifeste à l'égard des grands singes. Est-ce parce qu'ils présentent les capacités de conscience et de raison qui justifient, dans notre espèce, la reconnaissance du statut de patient moral ? Ou bien est-ce parce qu'il est plus facile d'éveiller l'empathie des humains en s'intéressant à l'espèce qui, du point de vue du sens commun, lui ressemble le plus ?

Si l'objectif est de faire valoir que les propriétés cognitives évoquées plus haut (langage, rationalité, conscience) justifient que l'on adopte un point de vue moral sur les organismes qui en sont pourvus, alors on ne voit pas pourquoi les grands primates seraient les seuls à bénéficier du statut de patient moral. Les dauphins satisfont beaucoup mieux qu'eux les critères avancés par Paola Cavalieri. Les dauphins (et les perroquets) sont bien meilleurs dans les tâches langagières que les chimpanzés ${ }^{16}$. Plus inventifs, plus flexibles devant la nouveauté, capables d'imitation et de mémorisation subtile de situations variées, les dauphins méritent certainement davantage l'attention des moralistes. Ils ne sont pas de notre lignée phylogénétique : mais en quoi la parenté directe forme-t-elle davantage un argument moral que la filiation dans une espèce ? Toute l'ambiguité de l'argumentation de Paola Cavalieri consiste à privilégier les comparaisons homologiques, et à reproduire avec la "préférence phylogénétique" les erreurs successives du racisme et de l'espécisme.

On peut à cet égard s'interroger sur le choix même des capacités retenues dans la sélection des espèces concernées par l'évaluation morale. Pourquoi l'existence de capacités quasi-langagières, produit accidentel de l'expérimentation humaine sur l'animal, devrait-elle être reconnue comme une caractéristique moralement pertinente ? Pourquoi le sens de soi-même, lié à l'organisation sociale et aux

16 Voir Vauclair, op. cit. 
différenciations qui y sont liées, devrait-il être tenu comme plus significatif que la capacité de se représenter l'environnement, d'apprendre à y repérer ce qui est utile ou nuisible ? Enfin la résolution de problème est en un sens une aptitude présente dans l'ensemble du monde animal, même si les moyens mis en oeuvre vont des dispositifs fixes et génétiquement transmis à des moyens flexibles et susceptibles d'être individuellement appris. Nous voyons ici une nouvelle résurgence du problème de la pente glissante. Sommes-nous condamnés à y succomber?

Le seul moyen de résister à cet argument, dans le cadre de la stratégie de la valeur intrinsèque adoptée par Paola Cavalieri, consisterait à justifier le point de vue comparatif non pas sur la base de ressemblances intuitives, mais sur la base de la compréhension fonctionnelle de ce qu'est un esprit. Avoir un esprit suppose la capacité de former des représentations portant sur le monde, c'est-à-dire d'avoir accès à l'information présente dans l'environnement en y distinguant des objets et événements, de la mémoriser et de la réutiliser. Un tel dispositif permet à l'individu qui en est pourvu de devenir l'agent de ses actions, de garder la trace de ses expériences successives et de répondre à des situations nouvelles sur la base de ses connaissances accumulées. Pourquoi, objectera-t-on ici, ce critère serait-il plus moralement fondé que les conditions évoquées par l'auteur ? Parce que c'est dans la mesure où un animal est capable de former des représentations que l'homme fait partie de son monde au même titre où l'animal fait partie du monde de l'homme. Peu importe ici que les représentations formées par les animaux des diverses espèces soient hétérogènes, diverses, sélectives. Peu importe que l'animal n'ait pas accès aux concepts dont dispose l'homme (et réciproquement). Il suffit que les conditions de la réciprocité cognitive soient présentes, au sens minimal où l'homme et les autres animaux partagent un monde où agir sur la base de leur expérience propre, entendons une expérience distinctive, irréductible, construite sous la pression de leurs besoins spécifiques. Cette capacité représentationnelle "transphylétique" justifie que l'on parle de communauté des esprits : en fait partie l'ensemble des animaux capables de former une représentation stable de leur univers, et d'utiliser leurs capacités mémorielles pour agir d'une manière flexible et rationnelle.

Les éthologistes et les philosophes continuent à débattre de l'existence d'une conscience animale, et d'une capacité sensitive chez les organismes les plus élementaires. Le critère proposé n'a pas à attendre la résolution de ce débat. Il existe en effet des critères cérébraux qui permettent d'identifier la capacité représentationnelle proprement mentale. Outre les capacités perceptives, et les aptitudes associatives requises par l'apprentissage, parfaitement testables, on peut 
supposer que l'une des conditions requises pour la catégorisation d'événements et d'objets consiste dans la présence d'un dispositif permettant à l'animal d'harmoniser les informations qui lui sont transmises par ses diverses modalités sensorielles, et de corriger éventuellement l'alignement de ses cartes sensorielles pour extraire une information spatiale cohérente ${ }^{17}$. Ce dispositif de recalibration est présent dans le cerveau des mammifères, des reptiles et des oiseaux, ce qui suggère d'étendre à ces animaux la communauté morale des esprits. Le choix de ce niveau d'analyse analogique a le mérite d'éviter l'exclusivisme (et l'anthopocentrisme latent) de la comparaison homologique: on peut avoir un esprit sans être un bipède, sans évoluer en milieu terrestre, et sans être membre d'un groupe social. Les conséquences du choix du nouveau critère sont certes autrement plus dévastatrices pour nos habitudes de carnivores, chasseurs et éleveurs que l'implication morale confinée au groupe des primates. Mais c'est le prix que doit payer l'espèce pourvue de réflexivité, qu'elle doive envisager de modifier ceux de ses apprentissages qui deviennent incompatibles avec le progrès de ses connaissances et le réajustement de ses valeurs.

17 Cf. J. Proust, Comment l'esprit vient aux bêtes, Paris, Gallimard, 1997. 